\title{
Assessment of the diagnostic accuracy of double inversion recovery sequence compared with FLAIR and T2W_TSE in detection of cerebral multiple sclerosis lesions
}

\author{
Zahra Abidi ${ }^{1}$, Fariborz Faeghi ${ }^{2}$, Zahra Mardanshahi ${ }^{3}$, Hasan Mortazavi $^{4}$
}

${ }^{1}$ M.Sc. Student of Medical Imaging Technology, Radiology Technology Department, School of Allied Medical Sciences, Shahid Beheshti University of Medical Sciences, Tehran, Iran

${ }^{2}$ Ph.D. of Medical Physics, Radiology Technology Department, School of Allied Medical Sciences, Shahid Beheshti University of Medical Sciences, Tehran, Iran

${ }^{3}$ M.D., Radiologist, Assistant Professor, Radiology Department, Mazandaran University of Medical Sciences, Sari, Iran

${ }^{4}$ M.D., Neurologist, Vali_E_Asr Hospital, Qaemshahr, Iran

Type of article: Original

\begin{abstract}
Background: Multiple sclerosis (MS) is a demyelinating disease of the central nervous system. MRI has an important role in early diagnosis of MS within diagnostic criteria.

Aim: To determine the diagnostic value of the double inversion recovery (DIR) sequence in detection of brain MS lesions.

Methods: In this cross-sectional study, 55 patients were admitted to the MRI department in Vali-E-Asr Hospital in Qaemshahr, Iran, from May 2016 to February 2016. Imaging was performed on a 1.5T Philips MR system using DIR, fluid attenuated inversion recovery (FLAIR), and T2-weighted turbo spin echo (T2W_TSE) sequences with the same parameters, including field of view (FOV), matrix, slice thickness, voxel size, and number of signal averaging (NSA). The DIR sequence has two different time inversions $\left(\mathrm{TI}_{1}=3400, \mathrm{TI}_{2}=325 \mathrm{~ms}\right)$ : suppressing cerebrospinal fluid (CSF) and white matter signal. Data analysis was performed using the SPSS version 20 , and $p$-value was gained from the patient-wise analysis by Wilcoxon analysis and paired samples t-test for matched pairs.

Results: More lesions in number and size were depicted on the DIR sequence compared with FLAIR $(\mathrm{p}=0.000$ with a relative ratio of 6 ) and T2W_TSE ( $\mathrm{p}=0.000$ with a relative ratio of 10). DIR demonstrated significantly more intracortical lesions compared with FLAIR ( $\mathrm{p}=0.000$ with a relative ratio of 2.53 ) and T2W_TSE ( $\mathrm{p}=0.000$ and relative ratio of 8.87). There was significantly higher contrast ratio between the white matter lesions and the normal appearing white matter (NAWM) in all anatomical regions especially in deep white matter $(\mathrm{p}=0.001)$.

Conclusion: An increasing total number of MS lesions can be detected by DIR sequence; thus, we recommend adding DIR sequence in routine MR protocols for MS patients.

Keywords: Double inversion recovery, FLAIR, T2W_TSE, MRI, Multiple sclerosis
\end{abstract}

\section{Introduction}

Recent studies have revealed gray matter (GM) involvement in the earliest clinical stages of multiple sclerosis (MS), which are correlations between the lesions and degree of physical and cognitive dysfunction. MRI plays an important role in early diagnosis of MS within diagnostic criteria. Consider the important diagnostic value of MRI in patients with clinically isolated syndromes (CIS), which may convert to definite MS followed by long-period disability and brain atrophy (1-6). While the disease distinctively affects the periventricular regions, calloso-septal interface, cerebellum, brainstem, and basal ganglia, nowadays more attention has been given to the function of the gray matter (7-9). Conventional MRI cannot demonstrate intracortical lesions (ICLs) well because of their small size

\section{Corresponding author:}

Dr. Fariborz Faeghi, Radiology Technology Department, School of Allied Medical Sciences, Shahid Beheshti University of Medical Sciences, Tehran, Iran.

Tel: +98.2122718506, Fax: +98.2122722150, Email: f_faeghi@sbmu.ac.ir

Received: August 30, 2016, Accepted: December 17, 2016, Published: April 2017

iThenticate screening: December 08, 2016, English editing: February 08, 2017, Quality control: March 20, 2017

(C) 2017 The Authors. This is an open access article under the terms of the Creative Commons Attribution-NonCommercialNoDerivs License, which permits use and distribution in any medium, provided the original work is properly cited, the use is non-commercial and no modifications or adaptations are made. 
and poor contrast compared with surrounding normal appearing gray matter (NAGM) as well as partial volume effects from cerebrospinal fluid (CSF) in T2-weighted spin echo/turbo spin echo (T2W_SE/TSE) and even in fluid attenuated inversion recovery (FLAIR) images (10-12). Several attempts were carried out recently, using different pulse sequences, to increase the sensitivity to detect more MS lesions in different anatomical regions. FLAIR imaging has less sensitivity in displaying lesions in the posterior fossa with the highest sensitivity near the CSF, like juxtacortical and the periventricular white matter. Despite the higher sensitivity of T2W_SE/TSE to display infratentorial lesions, the problem is to detect the juxtacortical lesions $(10,11,13)$. DIR images display great delineation of gray matter. The optimized contrast is due to differences in T1 relaxation times between GM/CSF and GM/WM (14-16). Redpath and Smith developed the DIR sequence, which produces dual contrasts of FLAIR and short time inversion recovery (STIR) $(15,17,18)$. Two combined inversion pulses in the DIR technique eliminates the signal from the normal appearing white matter (NAWM) and CSF and improves detection of cortical lesions (15, 19,20). The importance of gray matter damages in MS patients and the potential of DIR sequence to detect cortical lesions excited us to operate the technique and introduce the magnitude of DIR in many imaging centers in our country.

\section{Material and Methods}

In the present cross-sectional study, 55 patients ( 18 male, 37 female) were randomly selected from patients suffering from definite MS who were referred to the Neurology Department of Vali-E-Asr Hospital in Qaemshahr, Iran, from May 2016 to February 2016. Forty-two patients presented with RRMS (median age, 35.5 years; age range, 24-50 years) and 13 patients with SPMS (median age, 39 years; age range, 29-47 years). The present study was confirmed by the local ethics committee of the hospital, and all the patients went under examination after obtaining a written informed consent. Imaging was performed on a 1.5T Philips (Ingenia) MR system in the MRI Department of Vali-EAsr Hospital, Qaemshahr (Iran). Conventional MRI sequences were carried out as well as DIR, FLAIR, and T2W_TSE sequences. We compared these three sequences in the axial plane with identical anatomic position, using parameters including field of view (FOV), matrix, voxel size, number of signal averaging (NSA), and slice thickness and using a sensitivity encoding (SENSE) head coil. From our experience, the sagittal plane is preferred to display MS lesions in collo-septal and corpus callosum areas. But MS plaques in the areas of subcortical and juxtacortical are demonstrated better in axial orientation; thus, we decided to study MS plaques in the area by axial view. The parameters are noted in Table 1. The DIR sequence is known as GM only (gray matter only) and WM only (white matter only) imaging in Philips MR systems. An appropriate choice of inversion times allows either CSF and white matter to be suppressed, to depict the cortex alone, or CSF and gray matter to be suppressed, to depict the white matter. Two inversion delay times $\left(\mathrm{TI}_{1}=3400\right.$ and $\left.\mathrm{TI}_{2}=325 \mathrm{~ms}\right)$ are available in the system. First $\mathrm{TI}$ denotes intervals between the first $180^{\circ}$ inverter pulse and the $90^{\circ}$ excitation pulse, and the second TI denotes intervals between the second $180^{\circ}$ inverter pulse and the $90^{\circ}$ excitation pulse. It is necessary to lengthen the first TI in DIR compared with the TI in a FLAIR sequence for better CSF attenuation $(17,21)$.

Table 1. MRI sequence parameters

\begin{tabular}{|l|l|l|l|}
\hline Parameter & DIR & FLAIR & T2W_TSE \\
\hline Slice orientation & Axial & Axial & Axial \\
\hline Field of view (mm) & 230 & 230 & 230 \\
\hline Matrix & 256 & 256 & 256 \\
\hline Slice thickness (mm) & 5 & 5 & 5 \\
\hline Voxel size & 0.9 & 0.9 & 0.9 \\
\hline SENSE factor & 1.4 & 1.5 & 1.1 \\
\hline TSE factor & 17 & 53 & 16 \\
\hline Repetition time (ms) & 9600 & 10000 & 4500 \\
\hline Echo time (ms) & 25 & 140 & 100 \\
\hline Inversion time (ms) & $3400 / 325$ & 2800 & \\
\hline Number of signals averaged (NSA) & 2 & 2 & 2 \\
\hline Acquisition time (min:sec) & $2: 14$ & $3: 20$ & 3 \\
\hline
\end{tabular}

Note: DIR represents double inversion recovery; FLAIR, fluid-attenuated inversion recovery; T2 _TSE, T2weighted turbo spin-echo; SENSE, sensitivity encoding. First time inversion $\left(\mathrm{TI}_{1}=3400 \mathrm{~ms}\right)$ and second time inversion $\left(\mathrm{TI}_{2}=325 \mathrm{~ms}\right)$. Two saturation slabs were placed, one superiorly above the slices, on the sagittal sinus and the other one, inferiorly to the slices to eliminate blood vessels artifacts as well as switching on the flow compensation to decrease pulsation artifacts arising from large vessels. 


\subsection{Image analysis}

MS lesions appeared as hyper-intense spots with a size of $\geq 2 \mathrm{~mm}$. Contrary to these hyper-intense spots, there were some high signal intensity in a striped shape known as flow artifacts in extra cortical regions rising from the sinuses or major vessels. The number of lesions was calculated individually in each sequence, and lesions were divided according to their anatomical regions into (A) infratentorial lesions; (B) WM lesions; (C) intracortical lesions (ICLs). WM lesions are included to (a) periventricular lesions, which are adjacent to the lateral ventricles; (b) deep white matter (DWM) lesions, which are in deep WM, and (c) juxtacortical WM lesions, which actually are in the WM but touch the cortex $(2,15)$. The signal intensity was determined by locating the region-of-interest (ROI) in a mean size of $3 \mathrm{~mm} \times 3 \mathrm{~mm}$ on the lesions, the NAWM, and NAGM. The contrast ratios were calculated from the mean values of the contrast among lesions/NAGM, lesions/NAWM, lesions/CSF, and NAWM/NAGM separately in each technique and all anatomical regions. The contrast ratios were assessed according to $\left(\mathrm{SI}_{1} \mathrm{SI}_{2}\right) /\left(\mathrm{SI}_{1}+\mathrm{SI}_{2}\right)$, which $\mathrm{SI}_{1}$ representing the signal intensity of the lesions and $\mathrm{SI}_{2}$ representing the signal intensity of the NAWM, NAGM, or CSF. The contrast ratio between lesions and the NAWM were applied separately in infratentorial and supratentorial. Using the eight-element SENSE head coil and the automatic performing of the constant level appearance (CLEAR) algorithm in order to correct the signal intensity homogeneity for images made it difficult to carefully estimate signal-to-noise ratio (SNR) and contrast-to-noise ratio (CNR) $(2,17,22)$.

\subsection{Statistical analysis}

Total number of detected lesions and lesions in each region were separately determined and stated as mean \pm standard deviation and median. A Wilcoxon test was used for matched pairs to assess statistical differences in both analyses. The mean relative evaluation of detected lesions on DIR compared with FLAIR or T2W_TSE images was reported as a percentage gain or loss in the number of detected lesions. A positive relative measurement of lesions represented as a gain and a negative measurement represented a loss at DIR imaging regarding to FLAIR and T2W_TSE $(2,17,22)$. The contrast ratio differences between the sequences in different regions were distinguished using the Wilcoxon test for matched pairs. The statistical analysis was performed by the IBMC SPSSC Statistics version 20 (IBM@ Corp., Armonk, NY, USA) and $\mathrm{p}<0.05$ were presumed as statistically significant.

\section{Results}

In the present study, 2658 lesions were detected with DIR imaging, while 2513 lesions with FLAIR and 2423 lesions were detected with T2W_TSE. A significantly higher total number of lesions were displayed on DIR images compared with FLAIR ( $\mathrm{p}=0.000$ with a relative ratio of 6 ) and $\mathrm{T} 2 \mathrm{~W}(\mathrm{p}=0.000$ with a relative ratio of 10 ) imaging (Table 2). Lesions were depicted as being significantly higher on DIR imaging with respect to FLAIR and T2 weighted imaging in infratentorial region $(\mathrm{p}=0.000$ with a relative ratio of 18 and $\mathrm{p}=0.001$ and a relative ratio of 10 , respectively) (Table 2, Figure 1). A higher number of lesions in supratentorial regions in juxtacortical WM lesions was detected on DIR image compared with FLAIR ( $\mathrm{p}=0.000$ with a relative ratio of 8 ) and $\mathrm{T} 2 \mathrm{~W}$ ( $\mathrm{p}=0.000$ with a relative ratio of 18) images (Table 2, Figure 2). A slightly higher number of periventricular WM lesion was detected in DIR imaging with respect to FLAIR imaging ( $\mathrm{p}=0.14$ with a relative ratio of 1 ), but there was a significant difference between DIR and T2W images ( $\mathrm{p}=0.009$ with a relative ratio of 3 ) (Table 2). A relative loss of lesion detection at DIR imaging was observed regarding FLAIR and T2W imaging in the deep white matter series $(\mathrm{p}=0.285$ with a relative ratio of -2 and $\mathrm{p}=0.83$ with a relative ratio of -1 , respectively) (Table 2$)$. DIR imaging demonstrated significantly more intracortical lesions compared with FLAIR ( $\mathrm{p}=0.000$ with a relative ratio of 2.53) and T2W_TSE ( $\mathrm{p}=0.000$ with a relative ratio of 8.87 ) imaging (Figure 3,4$)$.
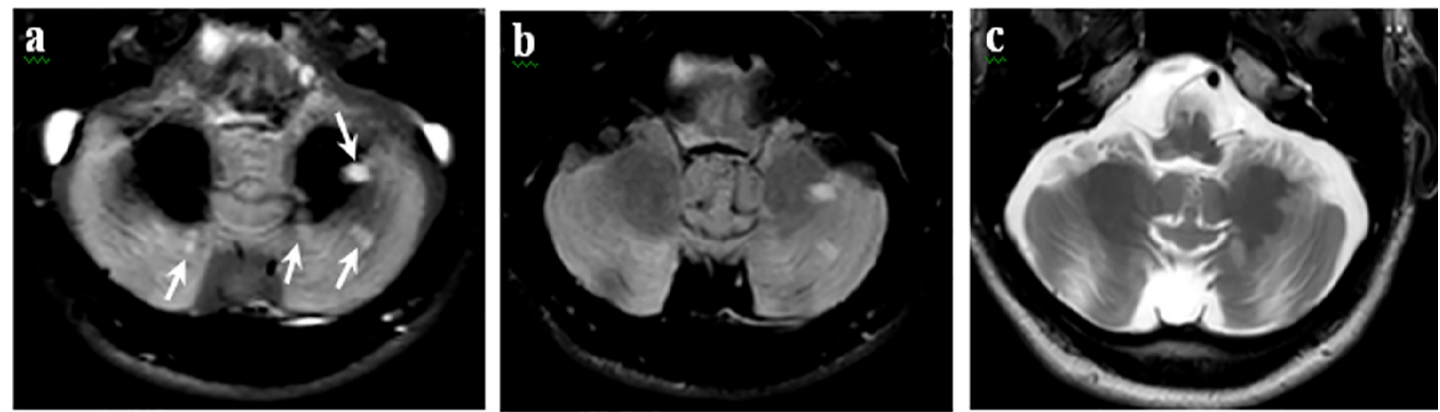

Figure 1. 36-year-old male with RRMS displays three lesions with better contrast and delineation in left cerebellum and a small plaque in the right hemisphere of cerebellum on (a) DIR image with no track on (b) FLAIR and (c) T2W images. 
Contrast measurements of DIR, FLAIR, and T2W_TSE imaging are listed in Table 3. There was a significantly higher contrast between the white matter lesions and NAWM on DIR images in infratentorial $(\mathrm{p}=0.039$ and $\mathrm{p}=0.017)$, periventricular $(\mathrm{p}=0.009$ and $\mathrm{p}=0.004)$ and juxtacortical $(\mathrm{p}=0.049)$ matched to both T2W TSE and FLAIR images. The contrast between DWM lesions and NAWM was prominent in DIR $(\mathrm{p}=0.001)$ compared with both T2W_TSE and FLAIR images. DIR sequences showed higher contrast ratio between the lesions and NAGM, when compared to $\mathrm{T} 2 \mathrm{~W}$ sequences $(\mathrm{p}=0.178)$, but there wasn't a statistical significance regarding to FLAIR imaging $(\mathrm{p}=0.626)$; however, the detected lesions by DIR were more clear. There was a significant difference between NAGM and NAWM in DIR imaging in comparison with T2W $(p=0.003)$ and FLAIR $(p=0.001)$ images. The contrast ratio between the lesions and CSF was significantly better regarding to T2W imaging $(\mathrm{p}=0.019)$ and was optimized with respect to FLAIR imaging without statistical significance $(\mathrm{p}=0.689)$.

Table 2. Analysis of the mean lesion load measurement and relative comparisons of DIR versus the FLAIR and T2WI sequences.

\begin{tabular}{|c|c|c|c|c|c|c|c|}
\hline \multirow[t]{2}{*}{ Region } & \multirow[t]{2}{*}{$\mathrm{DIR}^{\mathrm{a}}$} & \multirow[t]{2}{*}{ FLAIR $^{\mathrm{a}}$} & \multirow[t]{2}{*}{$\mathrm{T} 2 \mathrm{~W} \_\mathrm{TSE} \mathrm{a}^{\mathrm{a}}$} & \multicolumn{4}{|c|}{ Relative ratio $(\%)^{\mathrm{c}}$} \\
\hline & & & & $\begin{array}{l}\text { DIR/FLAIR } \\
(\%)\end{array}$ & $\begin{array}{l}P \\
\text { value }^{\mathrm{d}}\end{array}$ & $\begin{array}{l}\text { DIR/T2WI } \\
(\%)\end{array}$ & $\begin{array}{l}P \\
\text { value }^{\mathrm{d}}\end{array}$ \\
\hline Infratentorial & $\begin{array}{l}5.1 \pm 7.37 \\
(1)^{\mathrm{b}}\end{array}$ & $\begin{array}{l}4.23 \pm 6.45 \\
(0)^{\mathrm{b}}\end{array}$ & $\begin{array}{l}4.56 \pm 6.8 \\
(1)^{\mathrm{b}}\end{array}$ & 18 & $0.000^{*}$ & 10 & $0.001^{*}$ \\
\hline Periventricular & $\begin{array}{l}15.34 \pm 11.34 \\
(14)^{b}\end{array}$ & $\begin{array}{l}15.18 \pm 11.16 \\
(14)^{b}\end{array}$ & $\begin{array}{l}14.96 \pm 11.23 \\
(14)^{b}\end{array}$ & 1 & 0.14 & 3 & $0.009^{*}$ \\
\hline DWM & $\begin{array}{l}11.47 \pm 7.53 \\
(10)^{\mathrm{b}}\end{array}$ & $\begin{array}{l}11.69 \pm 7.75 \\
(10)^{\mathrm{b}}\end{array}$ & $\begin{array}{l}11.54 \pm 7.39 \\
(10)^{\mathrm{b}}\end{array}$ & -2 & 0.285 & -1 & 0.83 \\
\hline Juxtacortical & $\begin{array}{l}15.20 \pm 8.61 \\
(15)^{\mathrm{b}}\end{array}$ & $\begin{array}{l}14.07 \pm 8.11 \\
(13)^{\mathrm{b}}\end{array}$ & $\begin{array}{l}12.83 \pm 7.71 \\
(12)^{\mathrm{b}}\end{array}$ & 8 & $0.000^{*}$ & 18 & $0.000^{*}$ \\
\hline Intracortical & $\begin{array}{l}1.29 \pm 1.04 \\
(1)^{\mathrm{b}}\end{array}$ & $\begin{array}{l}0.5 \pm 0.71 \\
(0)^{\mathrm{b}}\end{array}$ & $\begin{array}{l}0.14 \pm 0.35 \\
(0)^{\mathrm{b}}\end{array}$ & 2.53 & $0.000^{*}$ & 8.87 & $0.000^{*}$ \\
\hline Total & $\begin{array}{l}48.32 \pm 27 \\
(41)^{b}\end{array}$ & $\begin{array}{l}45.69 \pm 25.57 \\
(41)^{b}\end{array}$ & $\begin{array}{l}44.05 \pm 24.8 \\
(41)^{\mathrm{b}}\end{array}$ & 6 & $0.000^{*}$ & 10 & $0.000^{*}$ \\
\hline
\end{tabular}

Note: DIR represents double inversion recovery; FLAIR: fluid-attenuated inversion recovery; T2W_TSE: T2weighted turbo spin echo. a) Data represent mean number of detected lesions \pm standard deviation; $\bar{b}$ ) median number of detected lesions; c) data indicate relative differences in number of detected lesions representing as the percentage of lesion numbers recognized by DIR imaging compared with matched FLAIR and T2WI; d) $p$-value was gained from the patient-wise analysis by Wilcoxon analysis and paired samples t-test for matched pairs, indicating that more or fewer patients showed higher lesion load measurement with DIR imaging compared with the corresponding FLAIR or T2WI.
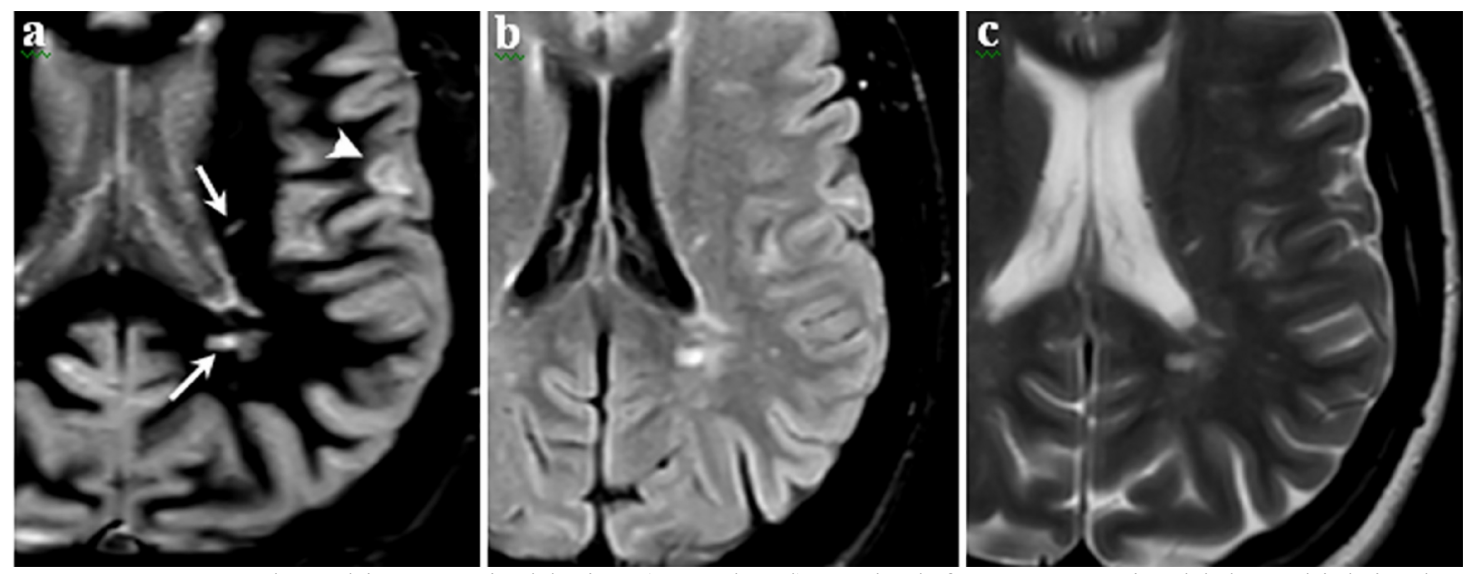

Figure 2. Worm-shaped juxtacortical lesion (arrowhead) on the left temporoparietal lobe, which is clear on (a) DIR image compared with (b) FLAIR and (c) T2W_TSE. Note to periventricular lesions (arrows) with better contrast on DIR image. 
Table 3. Image contrast measurement in DIR, T2W_TSE, and FLAIR images in different anatomical regions.

\begin{tabular}{|l|l|l|l|l|l|}
\hline Lesion/NAWM & DIR & T2WI & $P$ & FLAIR & $P$ \\
\hline Infratentorial & $0.67 \pm 0.15$ & $0.2 \pm 0.06$ & $0.039^{*}$ & $0.21 \pm 0.05$ & $0.017^{*}$ \\
\hline Periventricular & $0.85 \pm 0.12$ & $0.21 \pm 0.16$ & $0.009^{*}$ & $0.2 \pm 0.084$ & $0.004^{*}$ \\
\hline DWM & $0.93 \pm 0.036$ & $0.062 \pm 0.01$ & $0.001^{*}$ & $0.25 \pm 0.048$ & $0.001^{*}$ \\
\hline Juxtacortical & $0.79 \pm 0.19$ & $0.14 \pm 0.05$ & $0.049^{*}$ & $0.15 \pm 0.05$ & $0.049^{*}$ \\
\hline Lesion/CSF & $0.68 \pm 0.15$ & $0.006 \pm 0.01$ & $0.019^{*}$ & $0.6 \pm 0.17$ & 0.689 \\
\hline Lesion/NAGM & $0.14 \pm 0.039$ & $0.077 \pm 0.019$ & 0.178 & $0.077 \pm 0.19$ & 0.626 \\
\hline NAGM/NAWM & $0.68 \pm 0.01$ & $0.1 \pm 0.06$ & $0.003^{*}$ & $0.18 \pm 0.02$ & $0.001^{*}$ \\
\hline
\end{tabular}
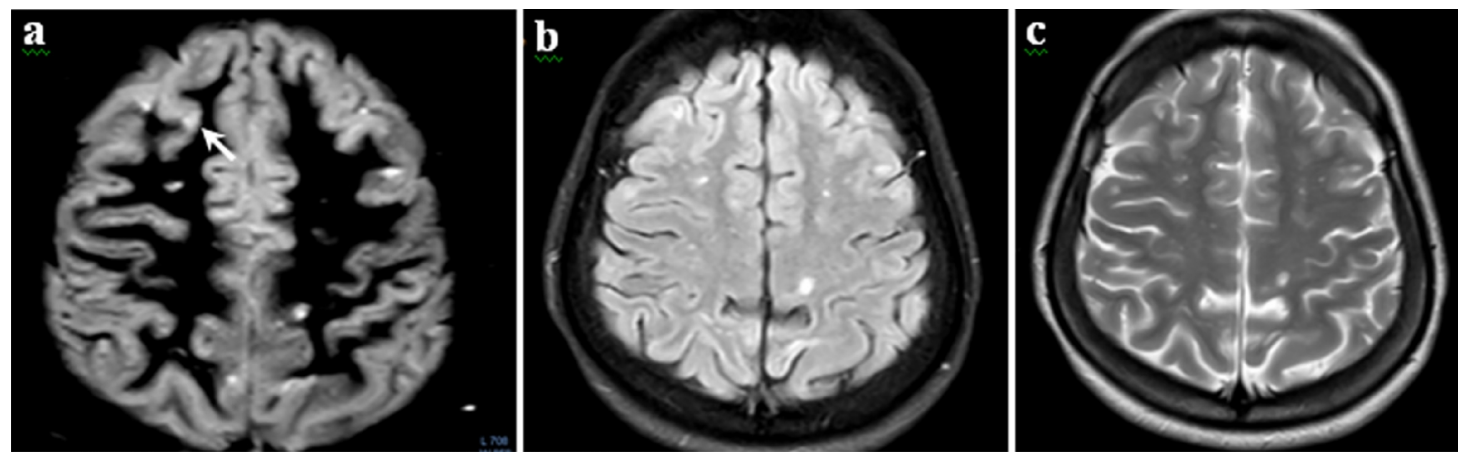

Figure 3. 36-year-old female with SPMS displayed numerous juxtacortical lesions in different regions with an intracortical lesion (arrow) in the cortex of right frontal lobe which is prominent in (a) DIR image compared with (b) FLAIR and (c) T2W_TSE.
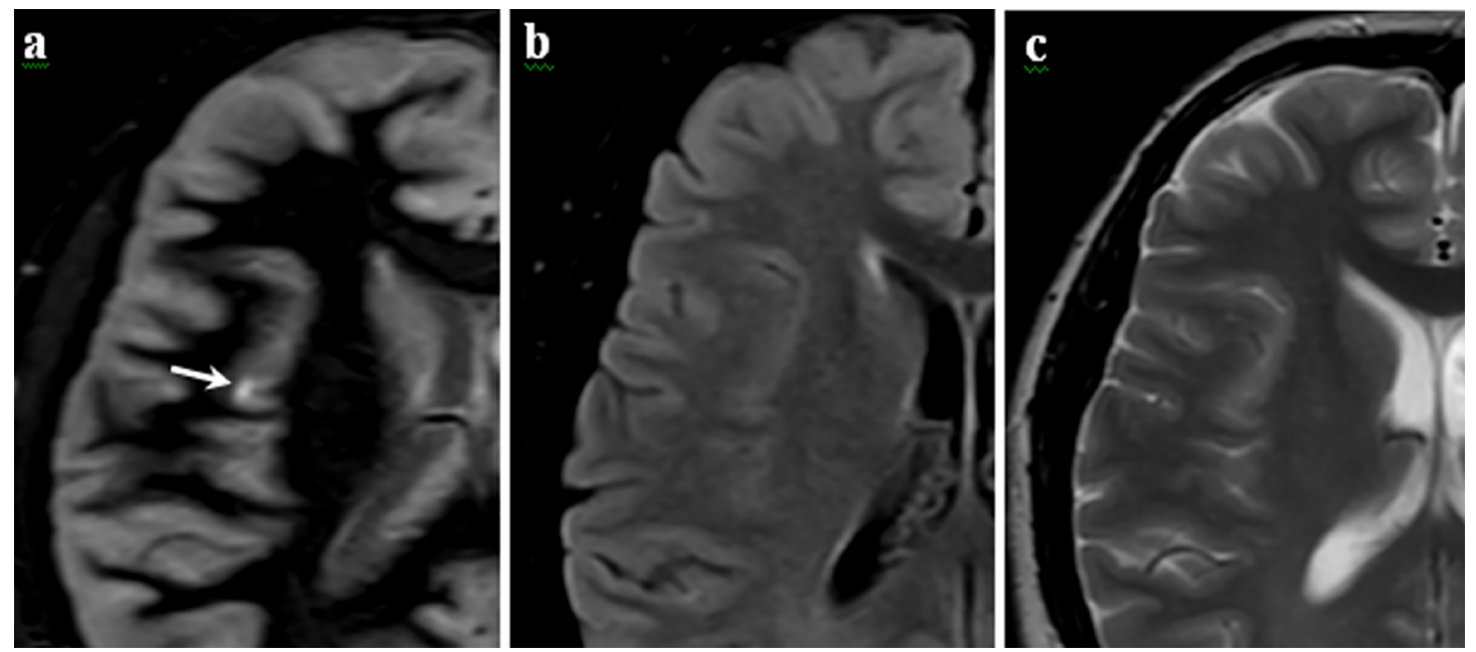

Figure 4. 36-year-old male with SPMS presented diplopia with an intracortical lesion in the cortex of right temporoparietal lobe on (a) DIR image, which is not seen on (b) FLAIR image and (c) T2_WI.

\section{Discussion}

Nowadays, contrary to new advanced MR techniques, including MR spectroscopy and diffusion tensor imaging (DTI) in patients with MS, conventional MRI sequences play an important role in early diagnosis of the disease in patients with CIS suggestive of MS or definite MS $(2,23)$. In a previous study, D. Chard acknowledged the role of DIR sequence for diagnostic criteria in patients with MS, especially for acute symptoms produced by new cortical lesions. GM damages in multiple sclerosis confirmed novel diagnostic criteria for these patients $(2,24,25)$. DIR showed a significantly higher total number of MS lesions in all anatomical regions compared with FLAIR and T2W_TSE, conforming to different studies $(2,15,17,22)$. Although T2W imaging is still considered the gold standard in infratentorial, we observed more lesions in comparison with FLAIR and T2W_TSE sequences in this region, which is equivalent with the result of Elnekeidy et al. (2), Geurts et al.(22), and Wattjes et al. (17) but contrary to the results of Vurtal et al. (15). ICLs were observed in about $74.5 \%$ of our patients by DIR sequence, 
$41.8 \%$ by FLAIR and $14.5 \%$ by T2W_TSE sequence. DIR sequence had the highest sensitivity and accuracy to detect intracortical lesions with respect to FLAIR and T2W_TSE sequences, which were equal to the results reported by Elnekeidy et al. (2), Geurts et al. (22), and Vural et al. (15). A significantly higher number of lesions in the supratentorial as seen in juxtacortical white matter, according to our study, Vural et al. (15), and Elnekeidy et al. (2) reported a significantly higher number of detected lesions in juxtacortical region on DIR images, but Geurts, J.J., et al. (22) reported the highest number of juxtacortical lesions on T2-weighted images. Wattjes et al. (17) noted slightly more lesions in the juxtacortical with FLAIR imaging. In the present study, DIR sequence demonstrated a slightly higher number of periventricular WM lesions in comparison with FLAIR but a statistical difference for $\mathrm{T} 2 \mathrm{~W}$ imaging, which was equivalent to the reported results from Wattjes et al. (17) and Geurts et al. (22) but inconsistent with the reported results from Elnekeidy et al. (2) and Vural et al. (15). A lower number of MS lesions were observed in deep white matter in the DIR sequence. Vural et al. (15) reported a higher number of detected lesions in DWM with T2W and FLAIR imaging regarding to DIR ( $\mathrm{p}=0.022$ and $\mathrm{p}=0.027$, respectively). FLAIR imaging displayed slightly more lesions in the DWM region compared with the DIR sequence, which are equivalent with the findings of Geurts et al. (22) and Wattjes et al. (17). But it is contrary with the finding of Elnekeidy et al. (2). In the present study, contrast measurements were calculated objectively in order to avoid creating subjective data by visual evaluation of contrast, which may be affected by windows and levels, magnification, and contrast and monitor brightness $(2,17)$. According to the main purpose of our study for lesion detection in these sequences, an improved distinction between GM/WM seems to be of second importance. The current study demonstrated better contrast ratio and delineation of the MS lesions on the DIR sequence compared with T2W_TSE and FLAIR sequences in all anatomical regions, which led to higher sensitivity to display MS lesions, especially ICLs. The contrast differences between the lesions and NAWM in FLAIR and T2W imaging were slightly equivalent in the infratentorial, periventricular, juxtacortical regions, but prominent in the DWM duo to completely suppress of WM in the DIR sequence. MS lesions near the CSF were more countable compared with confluent lesions in FLAIR and T2W_TSE because of better margins and contrast from great CSF nulling at DIR images. A better contrast ratio and improved distinction between NAGM/NAWM have been obtained on DIR imaging so, deciding about the location of the lesion in GM or WM was easier compared with FLAIR and T2W images. Some readers believed the interference of increased signals from GM with the high signal of MS lesions may make lesions less obvious on DIR in some patients; CSF nulling in some patients is less homogeneous on DIR images compared with FLAIR. However, CSF nulling was suitable on DIR images even in the worst conditions. Previous studies reported flow artifacts observed in posterior fossa, choroid plexus, periventricular WM, and periaqueductal, brainstem tissue, which may be from CSF pulsation or from sinuses and bigger vessels. Some other two-sided high-signal ribbon-like artifacts in the phase direction were often observed in extra-cortical regions, and their appearance change in continual sections. Also, lower SNR in the DIR technique and inhomogeneity of the magnetic field in the cortex of the limbic lobe and diminished inhomogeneity in the cortex of central sulcus should be noticed. Viewing multiple slices and other MRI sequences such as T1-weighted or FLAIR will help to report them as lesions (which are in an irregular shape) or as an artifact resulting from cortical vessels, which is rounded $(21,22,26,27)$. We arranged data acquisition by two saturation slabs: one superiorly placed above the slices on the sagittal sinus, and the other one inferiorly to the slices to eliminate blood vessels artifacts as well as switching on the flow compensation to decrease pulsation artifacts arising from large vessels; thus, we could obtain good results (Figure 5). Inflammation of new white matter lesions in MS is a cause for lesion enhancement on T1-weighted images after intravenous (IV) injection of a gadolinium-based contrast agents. T1 shortening is the eminent effect at common doses of gadolinium injection and contrast enhancement of active lesions causes to detect them on clinical MR images. Therefore, T1weighted-based sequences are usually used for post-contrast MR imaging. In addition to T2-weighted appearance on FLAIR images with dark cerebrospinal fluid, they have additional T1 contrast, which is the reason for the ability to see gadolinium-induced enhancement. As several papers have reported, FLAIR sequence has a high sensitivity to display MS plaques after gadolinium injection $(28,29)$. The aim of our study was to compare the number of detected lesions on these three sequences. Thus, it was performed on inactive MS lesions without IV contrast administration, and we came to the conclusion that DIR has higher sensitivity compared with FLAIR and T2W sequences. As previously mentioned, the contrast in DIR is a combination of STIR and FlAIR. Thus, there is hope that good results will come after injection. Despite the advantages of three-dimensional (3D) MRI such as improved detection of MS lesions, better spatial resolution, the ability of post-processing, and higher SNR over 2D sequences, oversampling in the slab direction will increase the acquisition time. 2D-DIR has superiority over 3D-DIR sequence due to higher acquisition time of 3D-DIR at high field strength such as $7 \mathrm{~T}$ and $3 \mathrm{~T}(2,11,30,31)$. The present study was limited to perform $2 \mathrm{D}$ pulse sequences due to the large number of referred patients to the imaging department as well as high scan time of 3D pulse sequences. Thus, it made us compare 2D DIR in optimal parameters with 2D FLAIR and 
2D T2W_TSE. As a limitation, there is no DIR (GM/WM only imaging) sequence in some MRI machines; thus, it is necessary to change some of the parameters in order to obtain DIR contrast.

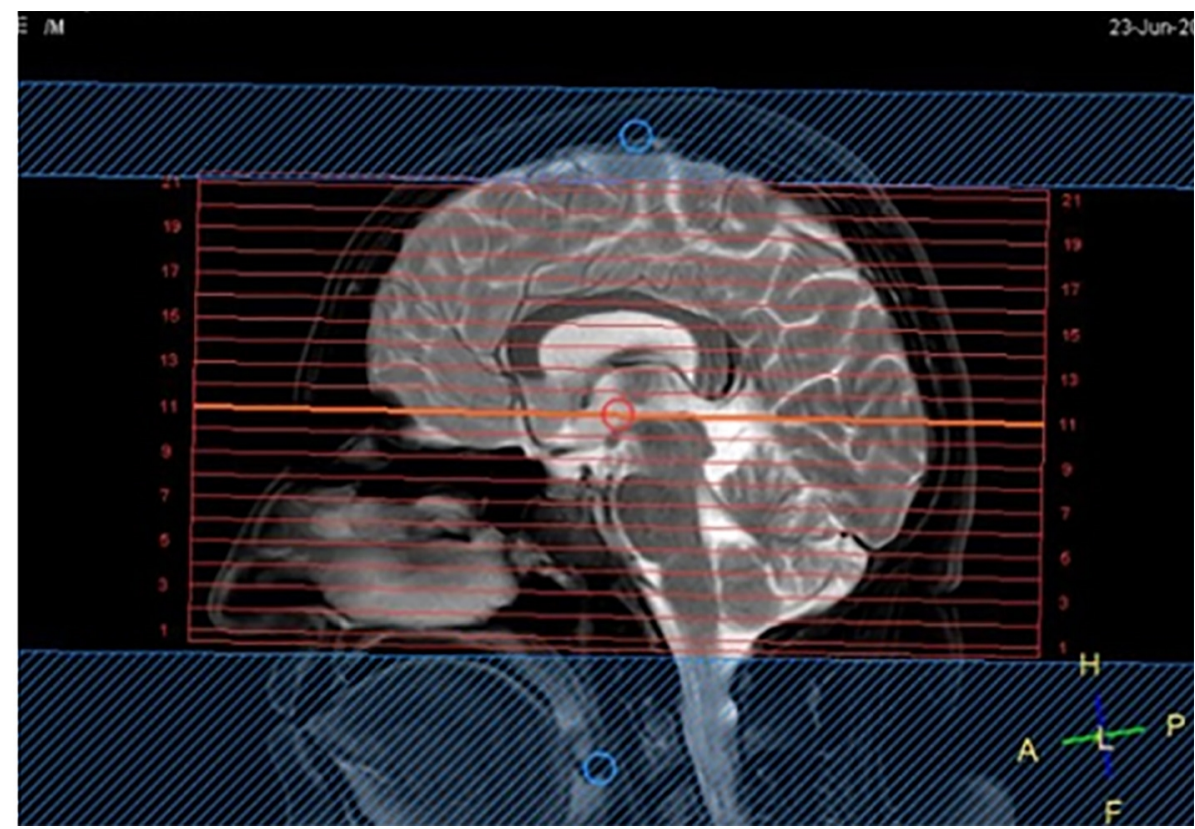

Figure 5. Using two saturation slabs, one superiorly over and another inferiorly under the slices to eliminate pulsation artifacts.

\section{Conclusions}

In conclusion, in the present decade MRI studies have revealed the importance of GM damage and its effect on a patient's disability. MRI has an important role in early diagnosis of multiple sclerosis, and CIS, which may convert to definite MS. Because the DIR sequence has higher sensitivity and accuracy in which to detect more MS lesions in number and size and display more intracortical lesions in comparison with routin sequences, we strongly recommend to add a DIR sequence in the routine MR protocols of MS patients.

\section{Acknowledgments:}

This is part of M.Sc. thesis supported by the Deputy of Research and Technology at Shahid Beheshti University of Medical Sciences. We would like to thank the patients and the staff of MRI department in Vali-E-Asr Hospital for the data acquisition; without their cooperation, this study would not have been possible. This research project was financially supported by Shahid Beheshti University of Medical Sciences.

\section{Conflict of Interest:}

There is no conflict of interest to be declared.

\section{Authors' contributions:}

All authors contributed to this project and article equally. All authors read and approved the final manuscript.

\section{References:}

1) Barkhof F, Rocca M, Francis G, van Waesberghe JH, Uitdehaag BM, Hommes OR, et al. Validation of diagnostic magnetic resonance imaging criteria for multiple sclerosis and response to interferon $\beta 1$. Annals of neurology. 2003;53(6):718-24. DOI: 10.1002/ana.10551. PMID: 12783417.

2) Elnekeidy AM, Kamal MA, Elfatatry AM, Elskeikh ML. Added value of double inversion recovery magnetic resonance sequence in detection of cortical and white matter brain lesions in multiple sclerosis. The Egyptian Journal of Radiology and Nuclear Medicine. 2014;45(4):1193-9. DOI: 10.1016/j.ejrnm.2014.06.010.

3) Minneboo A, Barkhof F, Polman CH, Uitdehaag BM, Knol DL, Castelijns JA. Infratentorial lesions predict long-term disability in patients with initial findings suggestive of multiple sclerosis. Archives of neurology. 2004;61(2):217-21. DOI: 10.1001/archneur.61.2.217. PMID: 14967769. 
4) Calabrese M, De Stefano N. Cortical lesion counts by double inversion recovery should be part of the MRI monitoring process for all MS patients: Yes. Multiple Sclerosis Journal. 2014;20(5):537-8. DOI: 101177/1352458514526084. PMID: 24692503.5.

5) Chen J, Narayanan S, Collins D, Smith S, Matthews P, Arnold D. Relating neocortical pathology to disability progression in multiple sclerosis using MRI. Neuroimage. 2004;23(3):1168-75. DOI: 10.1177/1352458514526084. PMID: 24692503.

6) Calabrese M, Rinaldi F, Mattisi I, Grossi P, Favaretto A, Atzori M, et al. Widespread cortical thinning characterizes patients with MS with mild cognitive impairment. Neurology. 2010;74(4):321-8. DOI: 10.1212/WNL.0b013e3181cbcd03. PMID: 20101038.

7) Lazeron RH, Langdon D, Filippi M, van Waesberghe JH, Stevenson V, Boringa JB, et al. Neuropsychological impairment in multiple sclerosis patients: the role of (juxta) cortical lesion on FLAIR. Multiple sclerosis. 2000;6(4):280-5. DOI: 10.1177/135245850000600410. PMID: 10962549.

8) Peterson JW, Bö L, Mörk S, Chang A, Trapp BD. Transected neurites, apoptotic neurons, and reduced inflammation in cortical multiple sclerosis lesions. Annals of neurology. 2001;50(3):389-400. DOI: 10.1002/ana.1123. PMID: 11558796.

9) Sormani MP. Modeling the distribution of new MRI cortical lesions in multiple sclerosis longitudinal studies by Sormani MP, Calabrese M, Signori A, Giorgio A, Gallo P, De Stefano N [PLoS One 2011; 6 (10): e26712. Epub 2011 October 20]. Multiple sclerosis and related disorders. 2012;1(3):108. DOI:10.1371/journal.pone.0026712. PMID: 22028937.

10) Geurts JJ, Barkhof F. Grey matter pathology in multiple sclerosis. The Lancet Neurology. 2008;7(9):841 51. DOI: 10.1016/S1474-4422(08)70191-1. PMID: 18703006.

11) Yousry TA, Filippi M, Becker C, Horsfield MA, Voltz R. Comparison of MR pulse sequences in the detection of multiple sclerosis lesions. American journal of neuroradiology. 1997;18(5):959-63. PMID: 9159377.

12) Filippi M, Rocca MA. MR imaging of multiple sclerosis. Radiology. 2011;259(3):659-81. DOI: 10.1148/radiol.11101362. PMID: 21602503.

13) Bakshi R, Ariyaratana S, Benedict RH, Jacobs L. Fluid-attenuated inversion recovery magnetic resonance imaging detects cortical and juxtacortical multiple sclerosis lesions. Archives of neurology. 2001;58(5):742-8. DOI:10.1001/archneur.58.5.742. PMID: 11346369.

14) Bedell BJ, Narayana PA. Implementation and evaluation of a new pulse sequence for rapid acquisition of double inversion recovery images for simultaneous suppression of white matter and CSF. Journal of Magnetic Resonance Imaging. 1998;8(3):544-7. DOI: 10.1002/jmri.1880080305. PMID: 9626866.

15) Vural G, Keklikoğlu H, Temel Ş, Deniz O, Ercan K. Comparison of double inversion recovery and conventional magnetic resonance brain imaging in patients with multiple sclerosis and relations with disease disability. The neuroradiology journal. 2013;26(2):133-42. DOI: 10.1177/197140091302600201. PMID: 23859234.

16) Redpath T, Smith F. Use of a double inversion recovery pulse sequence to image selectively grey or white brain matter. The British journal of radiology. 1994;67(804):1258-63. DOI: 10.1259/0007-1285-67-8041258. PMID: 7874427.

17) Wattjes M, Lutterbey G, Gieseke J, Träber F, Klotz L, Schmidt S, et al. Double inversion recovery brain imaging at 3T: diagnostic value in the detection of multiple sclerosis lesions. American journal of neuroradiology. 2007;28(1):54-9. PMID:17213424.

18) Ciccarelli O, Chen JT. MS cortical lesions on double inversion recovery MRI Few but true. Neurology. 2012;78(5):296-7. DOI: 10.1212/WNL.0b013e318245296f. PMID: 22218282.

19) Roosendaal S, Moraal B, Pouwels P, Vrenken H, Castelijns J, Barkhof F, et al. Accumulation of cortical lesions in MS: relation with cognitive impairment. Multiple Sclerosis. 2009;15(6):708-14. DOI: 10.1177/1352458509102907. PMID: 19435749.

20) Geurts J, Roosendaal S, Calabrese M, Ciccarelli O, Agosta F, Chard D, et al. Consensus recommendations for MS cortical lesion scoring using double inversion recovery MRI. Neurology. 2011;76(5):418-24. DOI: 10.1212/WNL.0b013e31820a0cc4. PMID: 21209373.

21) Turetschek K, Wunderbaldinger P, Bankier AA, Zontsich T, Graf O, Mallek R, et al. Double inversion recovery imaging of the brain: initial experience and comparison with fluid attenuated inversion recovery imaging. Magnetic resonance imaging. 1998;16(2):127-35. PMID: 9508269.

22) Geurts JJ, Pouwels PJ, Uitdehaag BM, Polman CH, Barkhof F, Castelijns JA. Intracortical Lesions in Multiple Sclerosis: Improved Detection with 3D Double Inversion-Recovery MR Imaging 1. Radiology. 2005;236(1):254-60. DOI: 10.1148/radiol.2361040450. PMID: 15987979. 
23) Simon J, Li D, Traboulsee A, Coyle P, Arnold D, Barkhof F, et al. Standardized MR imaging protocol for multiple sclerosis: Consortium of MS Centers consensus guidelines. American Journal of Neuroradiology. 2006;27(2):455-61. PMID:16484429.

24) Chard D. Cortical lesion counts by double inversion recovery should be part of the MRI monitoring process for all MS patients: no. Multiple Sclerosis Journal. 2014;20(5):539-40. DOI: 10.1177/1352458514526946. PMID: 24692504.

25) McDonald WI, Compston A, Edan G, Goodkin D, Hartung HP, Lublin FD, et al. Recommended diagnostic criteria for multiple sclerosis: guidelines from the International Panel on the diagnosis of multiple sclerosis. Annals of neurology. 2001;50(1):121-7. PMID: 11456302.

26) Pouwels PJ, Kuijer JP, Mugler III JP, Guttmann CR, Barkhof F. Human Gray Matter: Feasibility of SingleSlab 3D Double Inversion-Recovery High-Spatial-Resolution MR Imaging 1. Radiology. 2006;241(3):8739. DOI: 10.1148/radiol.2413051182. PMID: 17053197.

27) Calabrese M, De Stefano N, Atzori M, Bernardi V, Mattisi I, Barachino L, et al. Detection of cortical inflammatory lesions by double inversion recovery magnetic resonance imaging in patients with multiple sclerosis. Archives of neurology. 2007;64(10):1416-22. PMID: 17923625.

28) Absinta M, Vuolo L, Rao A, Nair G, Sati P, Cortese IC, et al. Gadolinium-based MRI characterization of leptomeningeal inflammation in multiple sclerosis. Neurology. 2015;85(1):18-28. DOI: 10.1212/WNL.0000000000001587. PMCID: PMC4501940.

29) Mathews VP, Caldemeyer KS, Lowe MJ, Greenspan SL, Weber DM, Ulmer JL. Brain: Gadoliniumenhanced Fast Fluid-attenuated Inversion-Recovery MR Imaging 1. Radiology. 1999;211(1):257-63. DOI: 10.1148/radiology.211.1.r99mr25257. PMID: 10189481.

30) Moraal B, Roosendaal SD, Pouwels PJ, Vrenken H, Van Schijndel RA, Meier DS, et al. Multi-contrast, isotropic, single-slab 3D MR imaging in multiple sclerosis. European radiology. 2008;18(10):2311-20. DOI: 10.1007/s00330-008-1009-7. PMID: 18509658.

31) de Graaf WL, Zwanenburg JJ, Visser F, Wattjes MP, Pouwels PJ, Geurts JJ, et al. Lesion detection at seven Tesla in multiple sclerosis using magnetisation prepared 3D-FLAIR and 3D-DIR. European radiology. 2012;22(1):221-31. DOI: 10.1007/s00330-011-2242-z. PMID: 21874361. PMCID: PMC3229693. 